\title{
A specific terminal structure is required for Ty1 transposition
}

\author{
D.J. Eichinger ${ }^{1}$ and J.D. Boeke \\ Department of Molecular Biology and Genetics, Johns Hopkins University School of Medicine, Baltimore, Maryland 21205 \\ USA
}

Yeast retrotransposon Ty1 directs the synthesis of virus-like particles (VLPs) consisting of Ty1-encoded proteins, RNA, and reverse transcripts. Ty1 reverse transcripts, tagged with a selectable marker and found within VLPs, are capable of transposing into naked target DNA in vitro. Cassettes consisting of a Ty long terminal repeat (LTR), or $\delta$, marked with supF, and flanked by appropriate restriction sites were constructed. These artificial substrates, whose termini resemble those of linear, full-length Ty1 reverse transcripts, can be coincubated with VLPs (containing unmarked reverse transcripts), resulting in the very efficient integration of the artificial substrate. The results suggest that Ty DNA is limiting for transposition in vivo, suggesting that inefficient reverse transcription regulates Ty1 transposition. Analysis of the transposition of these model substrates, which resemble in vivo Ty1 transposition intermediates or differ from them in subtle ways, shows that Ty transposition proceeds by the linkage of the $3^{\prime}$ hydroxyl residue of the reverse transcript to target DNA.

[Key Words: Saccharomyces cerevisiae; in vitro integration; reverse transcription; transposition]

Received October 13, 1989; revised version accepted December 18, 1989.

The Tyl element of Saccharomyces cerevisiae transposes to new sites using a mechanism akin to retroviral reverse transcription (Garfinkel et al. 1985; Mellor et al. 1985 ) and integration (Eichinger and Boeke 1988) processes. Tyl-encoded gene products and Tyl RNA assemble into a virus-like particle (VLP) resembling retroviral core particles (Garfinkel et al. 1985; Mellor et al. 1985). Small amounts of reverse transcriptase activity and free linear, double-stranded Ty1 DNAs (referred to as reverse transcripts in this paper) are also found in Tyl VLPs (Eichinger and Boeke 1988). An efficient tool for the study of Ty element transposition in vivo and in vitro is a family of recombinant plasmids called pGTy plasmids. These consist of the regulated yeast GAL1 promoter fused to a Ty element at its transcription initiation site, cloned in a high-copy-number $2 \mu$ plasmid (Boeke et al. 1985). The Ty element in one such plasmid, pGTy1-H3, can be marked by inserting foreign DNA into a nonessential site; such marked pGTy elements can transpose (Boeke et al. 1985, 1988).

Insertion of plasmid $\pi a n 7$, which carries the bacterial supF gene, into pGTyl-H3 results in a marked element whose transposition can be monitored both in vivo and in vitro. Transposition in vivo is monitored by determining the frequency of transposition into host chromosomal DNA per unit time (Boeke et al. 1985, 1988). In vitro transposition (i.e., the in vitro integration step of

${ }^{1}$ Present address: Department of Pathology, New York University Medical Center, New York, New York 10016 USA. the transposition process) is measured as follows. Tyl-H3- $\operatorname{an} 7$ VLPs are prepared from cells containing

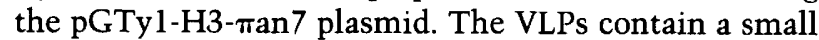
amount of preformed Tyl-H3- $\pi$ an 7 reverse transcript, primarily in the form of double-stranded linear DNA molecules, although other forms of Ty DNA [such as one long terminal repeat (LTR) circles] are also detected (Eichinger and Boeke 1988). The $\pi$ an7-supF-marked VLPs are incubated with target $\lambda g t \cdot W E S / \lambda B$ DNA and $\mathrm{Mg}^{2+}$. This $\lambda$ strain has amber mutations in three essential genes. The reaction mixture is then deproteinized, and the nucleic acid mixture is packaged into $\lambda$ phage heads. Transposition of Ty1-H3- $\tan 7$ into nonessential portions of the $\lambda$ genome results in recombinant phage $\lambda$ derivatives able to form plaques on nonsuppressing bacterial host strains. Transposition is quantified by comparing the number of recombinant (supF-containing) phage (number growing on the nonsuppressing host) to the total number of phage in the final packaging reaction (number growing on a suppressor-containing host). The in vitro reaction requires only the particulate Ty VLP fraction; there is no direct evidence that soluble macromolecular factors participate in the reaction (Eichinger and Boeke 1988). (It is formally possible that non-Ty products contaminating the VLPs participate in the integration reaction.) Ty VLPs have structural and functional properties that are very similar to those of particulate Moloney murine leukemia virus nucleoprotein complexes isolated from infected cells (Brown et al. 1987, 1989; Fujiwara and Mizuuchi 1988; Bowerman et al. 1989; Fujiwara and Craigie 1989|. 


\section{Results}

\section{Artificial substrates for in vitro Ty1 transposition}

We are studying the Tyl integration process with defined components by developing a set of cloned substrates consisting only of terminal Ty LTR (or $\delta$ ) sequences flanking a plasmid, $\pi a n 7$, bearing the selectable marker supF. These substrates are generated by cutting with various restriction enzymes whose recognition sites flank the LTR sequences and then modifying the restriction enzyme-generated termini further with other enzymes. Two such substrates have been constructed, using polymerase chain reaction (PCR), and cloned in M13 vectors (for details of the constructions, see Materials and methods). The structures of M13 inserts $\delta \pi 1$ (flanked by FokI and NsiI sites) and $\delta \pi 2$ (flanked by HindIII sites), from which a family of substrates can be generated, are shown in Figure 1. The $\delta \pi 1$ insert can be converted to a blunt-ended substrate molecule $\delta \pi l-0$, whose terminal structure corresponds to that of a full-length

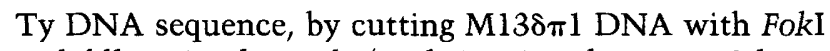
and filling in the ends (with DNA polymerase I large fragment). A molecule of identical structure can be generated by cutting $\mathrm{M} 13 \delta \pi 1$ with $N$ siI (or M13 $\delta \pi 2$ with $H$ indIII) and treating with S1 nuclease.

$\delta \pi$ 1-0 molecules, when mixed with Tyl VLPs /which lack a selectable marker, themselves) and target $\lambda$ DNA, are efficiently transposed into the $\lambda$ DNA in vitro, presumably by a reaction requiring the transposon-encoded IN (integrase) protein. The VLP-mediated reaction using this exogenous substrate has the same very simple biochemical requirements of the reaction based on marked

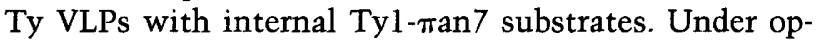
timal conditions, at least $0.09 \%$ of this exogenous $\delta \pi 1-0$ substrate is converted into recombinant molecules (calculated as indicated in Materials and methods|. It is also instructive to consider the efficiency of the reaction in terms of how many cell-equivalents of VLPs are required to give rise to each in vitro transposition event. When 50 $\mathrm{ng}$ of $\delta \pi 1-0$ substrate (a saturating amount) is mixed

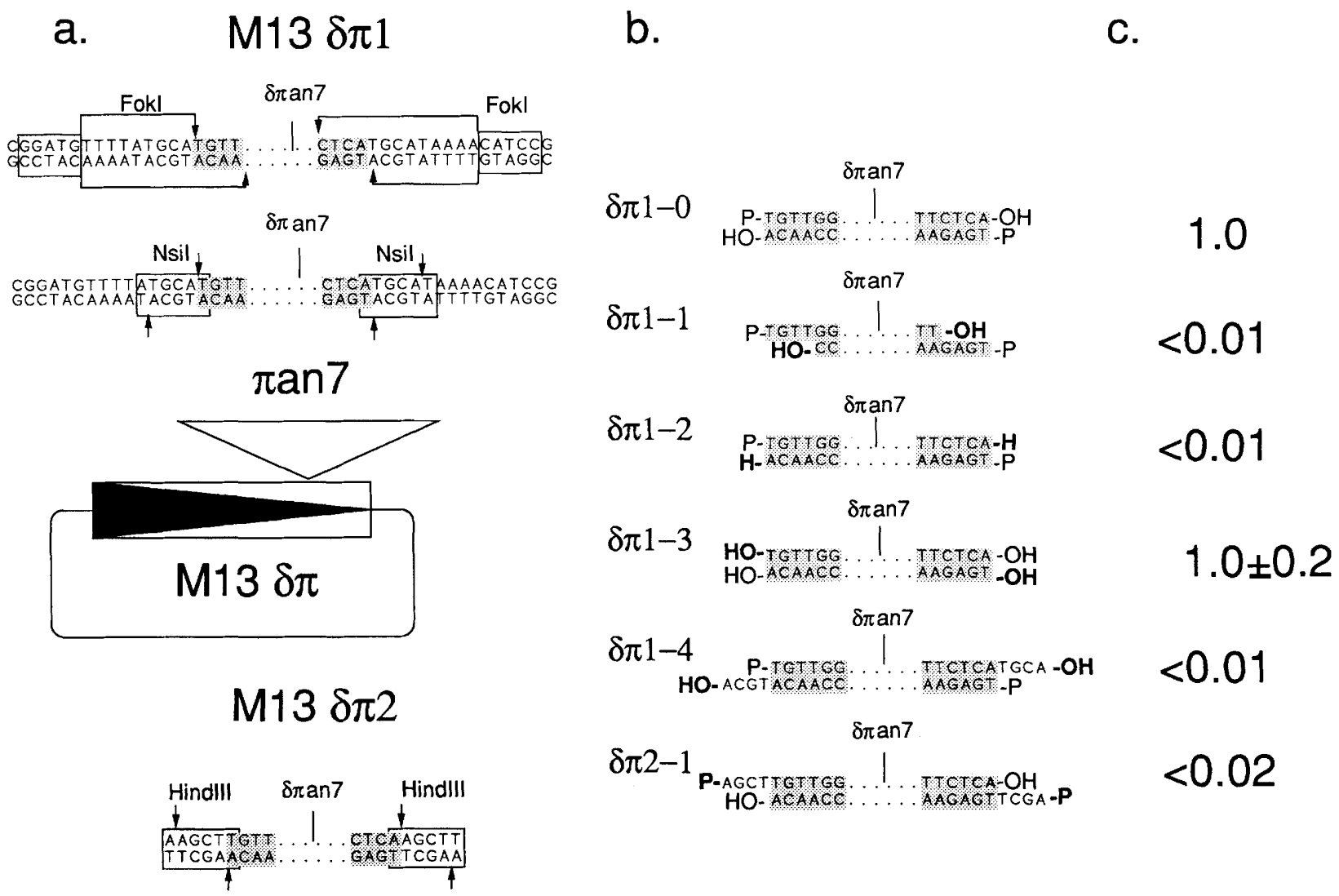

Figure 1. Structure and transposition of recombinant M13 phage DNA and derived $\delta \pi$ substrates. $(a)$ The diagram indicates the

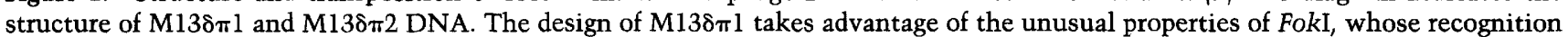
and cut sites are different. The boxed triangle indicates LTR or $\delta$ sequence. The sequences above and below the diagram show the relevant sequences surrounding the $\delta \pi a n 7$ sequence. $\delta$ sequences are shaded. Restriction enzyme recognition sites are boxed; cleavage

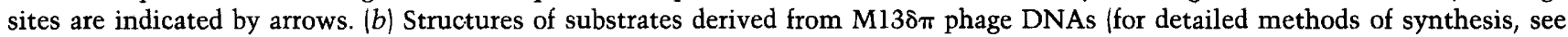
Materials and methods). Terminal functional groups are indicated in boldface when different in structure or position from those in

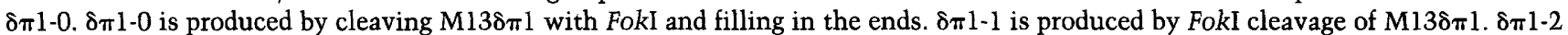
differs from $\delta \pi 1-0$ in that it lacks a $3^{\prime}$-hydroxyl group (the terminal adenosines were incorporated in the form of $2^{\prime}, 3^{\prime}$-ddATP). $\delta \pi 1-3$

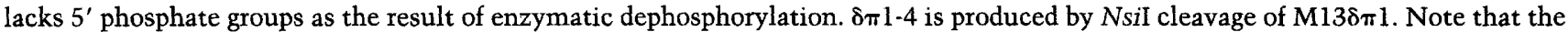

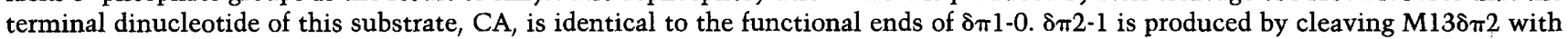
HindIII. (c) The effectiveness of these substrates in the in vitro transposition reaction (relative to $\delta \pi 1-0$ ) is indicated. 
Figure 2. Structure of termini of in vitro integration events. The 5-bp duplication of Ty sequences is indicated by shading. Sites of insertion into $\lambda$ are $\lambda d p-1,23376 ; \lambda d p-3,47218 ; \lambda d p-9,23677$ (Sanger et al. 1982).

with VLPs derived from $\sim 3.5 \times 10^{7}$ cells under ideal conditions, then $3.4 \times 10^{7}$ transposition events result. Thus, a one-cell equivalent of VLPs gives rise to one transposition event. This efficiency, however, because the efficiency varies from preparation to preparation and presumably reflects the ability to recover is presumably a minimum estimate of the "active" VLPs.

\section{In vitro transposition events have the expected structure}

Restriction mapping of $10 \lambda / \delta \pi$ recombinant phage was performed; the digests were all consistent with simple insertion of $\delta \pi$ into $\lambda$. Nine of these were unambiguously mapped within the $\lambda$ genome, and three were sequenced on both ends. The expected 5-bp duplication of $\lambda$ sequence was found at the termini of each $\delta \pi$ insert (Fig. 2).

\section{Ty DNA is limiting for transposition}

Unlike the reaction requiring internal Ty- $\tan 7$ substrate, the reaction utilizing exogenous $\delta \pi$ substrates can be sustained over long time periods (Fig. 3). Whereas the reaction utilizing internal Tyl-man 7 DNA reaches a maximum within several minutes, the $\delta \pi 1-0$-dependent reaction is nearly linear for at least $1 \mathrm{hr}$. This result suggests that the amount of DNA with appropriate terminal structures inside the Ty-VLPs is limited. This interpretation is also supported by our inability to observe conversion of a significant fraction of the Ty DNA found

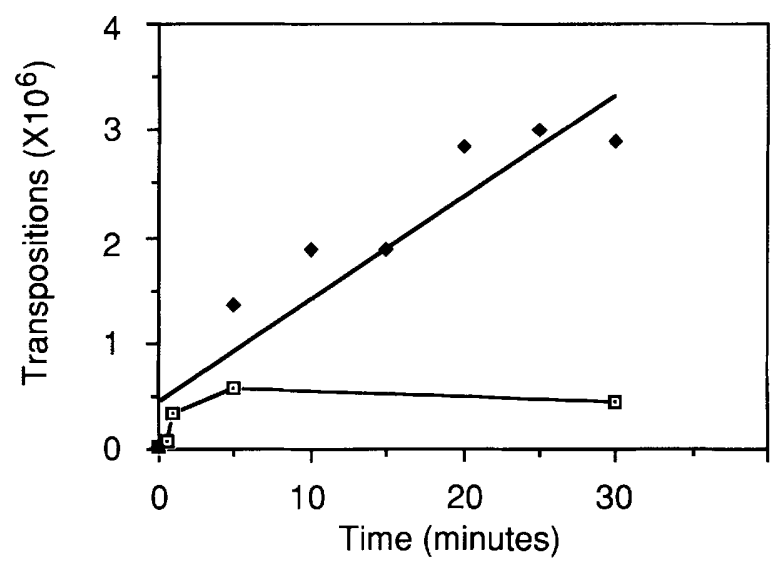

Figure 3. Time courses of in vitro transposition reactions. Aliquots were withdrawn from the reaction, as indicated. (ㅁ) Reaction using VLPs containing $\pi$ an 7 -marked reverse transcripts, prepared from strain JB619. $\$$ Reaction using VLPs containing unmarked reverse transcripts, prepared from strain JB224, and $\delta \pi 1-0$ DNA.

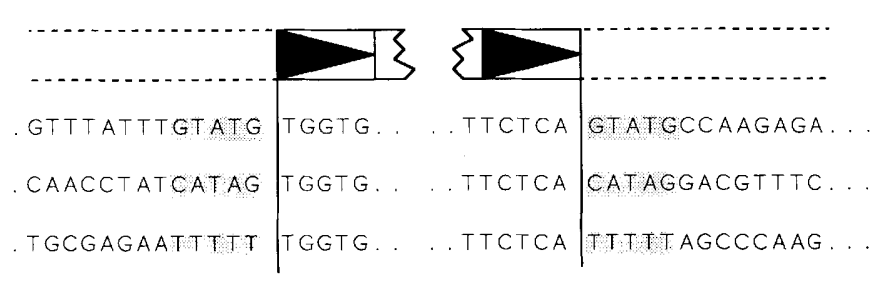

inside particles into recombinant molecules. [To detect such recombinants, we used a Southern blotting approach similar to that used by others studying retrovirus integration (Fujiwara and Mizuuchi 1988; Brown et al. 1989; data not shown)]. Such high-efficiency conversion of endogenous reverse transcripts to recombinant form has been reported for the in vitro reaction of Moloney murine leukemia virus (MuLV) nucleoprotein particles, in which $10-25 \%$ of the reverse transcripts integrate into target DNA. We estimate that the Tyl integration reaction utilizing internal substrate incorporates only $0.1-0.3 \%$ of the reverse transcripts in the VLPs into target DNA (Eichinger and Boeke 1988). We tested whether the in vitro integration reaction utilizing endogenous reverse transcripts is limited by non-nucleic acid components required for integration by adding $\delta \pi 1-0$ DNA to such a reaction. Addition of exogenous artificial substrate results in $\sim 100$-fold more in vitro transposition events (Table 1 ).

We also made direct measurements of the total amount of Ty DNA in Tyl-VLP preparations (from cells bearing induced pGTyl-H3 plasmids). Remarkably, the VLPs in these cells contain only $\sim 10$ full-length molecules of unintegrated Ty DNA per cell (Table 2).

\section{Structural requirements for the in vitro integration reaction}

Because the integration reaction occurs under conditions that prevent DNA synthesis, it is presumed that the immediate product of integration is a recombinant molecule with a 5-bp gap in the target DNA at each end of the $\delta \pi$ sequences; these gaps could be at either the $5^{\prime}$ or $3^{\prime}$ ends of $\delta \pi$. Several variant forms of the $\delta \pi$ substrate have been constructed to evaluate the reaction requirements at the level of the Tyl substrate.

We generated a family of analogs of the efficient trans-

Table 1. Transposition of endogenous reverse transcripts is limited by the amount of available substrate

\begin{tabular}{|c|c|c|}
\hline $\begin{array}{l}\text { Source of } \\
\text { Ty VLPs }\end{array}$ & $\begin{array}{l}\delta \pi 1-0 \text { added } \\
\text { (ng) }\end{array}$ & $\begin{array}{l}\text { Total marked } \\
\text { transposition events }\end{array}$ \\
\hline $\mathrm{JB} 224^{\mathrm{b}}$ & 0 & $<6.0 \times 10^{3}$ \\
\hline JB224 ${ }^{b}$ & 50 & $3.4 \times 10^{7}$ \\
\hline JB619c & 0 & $1.4 \times 10^{5}$ \\
\hline JB619c & 50 & $1.3 \times 10^{7}$ \\
\hline
\end{tabular}

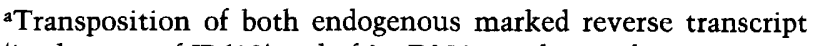
(in the case of JB619) and of $\delta \pi$ DNA are detected.

bContains unmarked Ty reverse transcripts (Boeke et al. 1985; Eichinger and Boeke 1988).

cContains $\pi$ an7-marked Ty reverse transcripts (Eichinger and Boeke 1988). 
Table 2. DNA content of Ty VLPS

\begin{tabular}{llcc}
\hline Strain & $\begin{array}{l}\text { Number } \\
\text { of cells }\end{array}$ & $\begin{array}{l}\text { Tyl DNA } \\
(\mathrm{ng})\end{array}$ & Copies per cell \\
\hline JB619 & $1.7 \times 10^{9}$ & 100 & 7.8 \\
JB224 & $1.4 \times 10^{9}$ & 130 & 14.7 \\
\hline
\end{tabular}

aAmount of DNA recovered from VLP preparation; determined by quantitative Southern blotting with plasmid DNA standards.

position substrate $\delta \pi 1-0$ by a variety of enzymatic methods (for details, see Materials and methods). These analogs were then compared to $\delta \pi 1-0$ for in vitro integration efficiency. The substrates tested fell into two classes: (1) inactive as a transposition substrate (transposing at frequencies at least 100-fold lower than $\delta \pi 1-0$ itself), or (2) about as effective as $\delta \pi 1-0$ itself (Fig. 1). Substrate $\delta \pi 1-1$ is produced by digestion with FokI but without filling in; it is inactive in transposition. Substrate $\delta \pi 1-2$, which is identical to $\delta \pi 1-0$, except that it lacks a 3 '-hydroxyl group, is inactive for transposition. This substrate is prepared by filling in the last adenylate residues using $2^{\prime}, 3^{\prime}$-dideoxyadenosine triphosphate rather than 2 '-deoxyadenosine triphosphate. Substrate

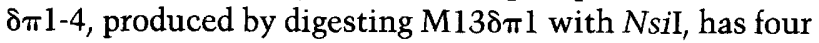
extra nucleotides on the $3^{\prime}$ end of the substrate; it is inactive as a substrate for transposition. Furthermore, removal of the $5^{\prime}$ phosphate of substrate $\delta \pi 1-0$ with alkaline phosphatase produces a substrate, $\delta \pi 1-3$, fully active in transposition, indicating that the $5^{\prime}$ phosphate groups of the transposon are dispensable for transposition. These results strongly support the idea that the $3^{\prime}$ hydroxyl end of the $\delta \pi$ substrate is joined to the $5^{\prime}$ end of a staggered break in target DNA and that the $5^{\prime}$ ends of $\delta \pi$ and the $3^{\prime}$ ends of target DNA flank the gaps. Substrate $\delta \pi 2-1$, produced by digestion of $M 13 \delta \pi 2$ with HindIII, is inactive as a substrate. Apparently, although no $5^{\prime}$ phosphate is required for $\delta \pi$ transposition, the presence of four extra deoxyribonucleosides at the $5^{\prime}$ end of the substrate blocks $\delta \pi$ transposition effectively. This is surprising because in natural Tyl reverse transcripts, the $5^{\prime}$ end (of the "bottom" strand) is thought to be formed by extension of RNA primers such as initiatormethionine tRNA (Eibel et al. 1981; K. Chapman, A. Bystrom, and J.D. Boeke, unpubl.). The hindrance of the in vitro reaction by extra DNA bases suggests that such RNA primers must be removed prior to formation of ends active in transposition.

\section{A covalently closed circular substrate is inactive in transposition}

We generated a covalently closed circular form of $\delta \pi 1-0$, as well as concatemers of $\delta \pi 1-0$, by ligation of $\delta \pi 1-0$ monomers with T4 DNA ligase (Fig. 4). These derivatives of $\delta \pi 1-0$, as well as unreacted $\delta \pi 1-0$, were eluted from an agarose gel and used as substrates in the in vitro integration reaction. Whereas $\delta \pi 1-0$ (and its concatemerized forms; data not shown) readily gave rise to recombinants, none were detected with the covalently closed circular material, indicating that it is at least 50fold less active in transposition than the corresponding monomer linear substrate.

\section{Only terminal Ty1 sequences are required for efficient integration}

We constructed two deletion derivatives of $M 13 \delta \pi 1$,

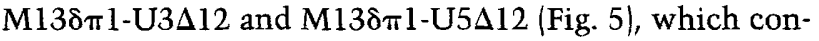
tain only $12 \mathrm{bp}$ of Tyl sequence at the $5^{\prime}$ and $3^{\prime}$ ends of the LTR, respectively. FokI treatment, followed by filling in, converts both of these mutant DNAs into active substrates. The activities of these substrates are within twofold of the activity of $\delta \pi 1-0$. Similarly, the

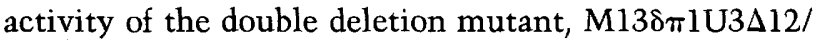
U $5 \Delta 12$, containing only $24 \mathrm{bp}$ of Ty 1 sequence, is within twofold of $\delta \pi 1-0$ (Table 3). Thus, the transposition machinery recognizes a region of $<13$ bp at each terminus of the $\delta \pi$ substrate molecule.

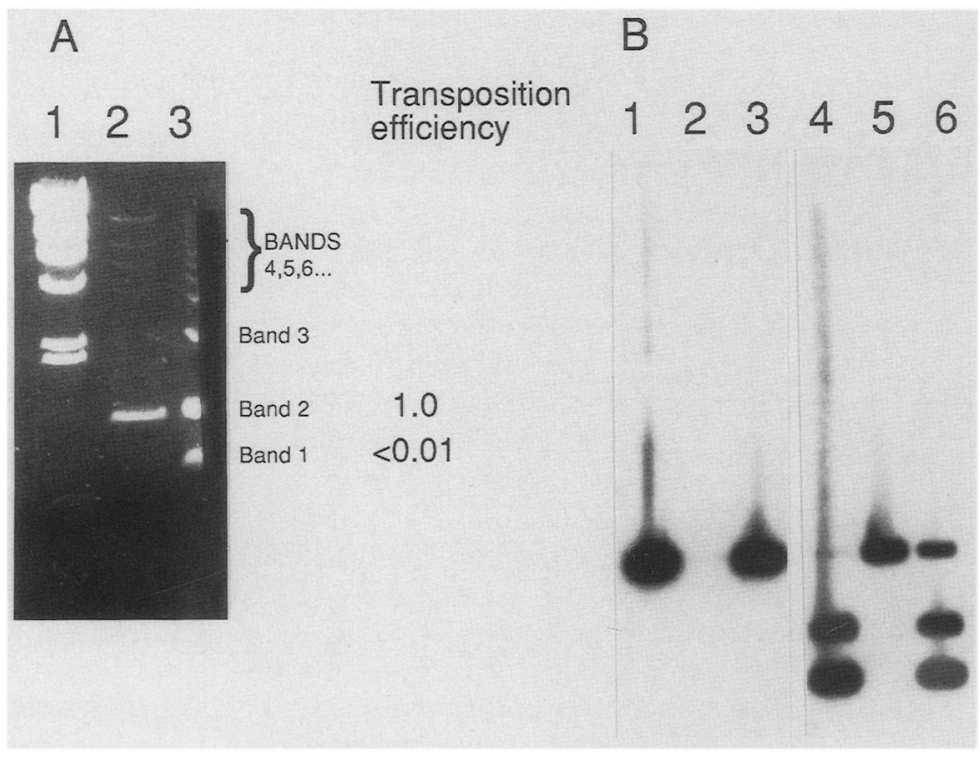

Figure 4. Ligation products of $\delta \pi 1-0$ and their transposition. (A) Preparative ethidium-bromide-stained agarose gel. (Lane 1) Molecular weight markers; (lane 2) unligated $\delta \pi l-0$; (lane 3$)$ ligated $\delta \pi l-0$. Indicated bands were purified by electroelution and used for in vitro transposition. The relative efficiency of transposition, per molecule of input $\delta \pi$ substrate, normalized to $\delta \pi 1-0$, is indicated for bands 1 and $2 .(B)$ DNA eluted from bands 1 and 2 was re-electrophoresed with (lanes 4-6) and without (lanes 1-3) prior DdeI digestion. There is one DdeI site in $\delta \pi l-0$. The gel was transferred to nitrocellulose and hybridized with a Tyspecific probe. Based on their behavior following DdeI digestion, the bands were identified as follows: (band 1) Monomeric circles; (band 2) monomeric linears (may also contain some dimeric circles); (band 3) dimeric linears; (bands 4 and up) multimeric linears. (Lanes 1 and 4) Unligated $\delta \pi 1-0$; (lanes 2 and 5) band $\mathbf{l}_{\text {; }}$ (lanes 3 and 6) band 2. Band 1 material could only be detected after cleavage (cf. lanes 2 and 5). 
$\mathrm{U} 3 \Delta 12$ TGT F GGAAT AGAGGTCACCAGCTTGCGTTGCTGG ACAACETT A TOT CCAGTGGTCGAACGCAACGACC BstEll

\section{U $5 \Delta 12$}

$\operatorname{\pi an} 7$

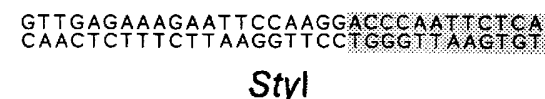

Figure 5. Structure of deletion mutants. Mutant derivatives of $M 13 \delta \pi 1$ were constructed by oligonucleotide-directed mutagenesis (Kunkel 1985). Tyl-derived sequences are shaded. Unique restriction sites incorporated into the oligonucleotides are indicated.

\section{Discussion}

Tyl reverse transcripts are found in the form of a nucleoprotein complex, the VLP (Eichinger and Boeke 1988). A similar structure can be isolated from cells infected with the Moloney MuLV (Brown et al. 1987; Bowerman et al. 1989). These structures contain all of the macromolecules needed to effect in vitro integration. Our studies and related studies of Moloney virus integration (Fujiwara and Craigie 1989) show that the reverse transcript can be provided as a cloned molecule whose termini resemble those of native reverse transcripts; apparently, the integration machinery can recognize these soluble molecules and, in the case of Tyl at least, can integrate them very efficiently. Whether the joining reaction takes place within the VLP (where the IN protein presumably resides) or outside the VLP structure is uncertain. However, we note that nucleases and substrates for reverse transcriptase are able to access the Ty-encoded reverse transcriptase without detergent treatment or other obvious insult to the VLPs (Garfinkel et al. 1985; Mellor et al. 1985). Thus, it is not unlikely that $\delta \pi 1-0$ DNA can enter Tyl VLPs. By manipulating the functional groups and DNA sequences at the termini of these molecules, we have established that the Tyl integration reaction (1) works efficiently using a bluntended substrate, $(2)$ requires the 3 '-hydroxyl group of the terminal adenylate residues, $(3)$ does not require $5^{\prime}$ phosphates, and (4) recognizes sequences within the terminal $12 \mathrm{bp}$ of the reverse transcript.

That the Tyl integration reaction uses a blunt-ended substrate differs from the situation in retroviruses, where a substrate with a 2-bp gap at the $3^{\prime}$ end, which is generated by IN protein (Brown et al. 1989; Roth et al. 1989|, is used. This difference is perhaps not too surprising when the slight differences in sequence organization between Tyl and retroviruses is considered. The position of the putative primer binding site for Ty reverse transcription of the $|-|$ strand (relative to the LTR sequence) is offset from that of retrovinuses by $2 \mathrm{bp}$ (Fig.

Table 3. Transposition of deletion mutant substrates

\begin{tabular}{lc}
\hline Substrate & Number of transpositions $(50 \mathrm{ng}$ DNA) \\
\hline$\delta \pi 1-0$ & $3.7 \times 10^{6}$ \\
$\delta \pi 1-0$ U3 $\Delta 12$ & $3.4 \times 10^{6}$ \\
$\delta \pi 1-0$ U5 $\Delta 12$ & $1.7 \times 10^{6}$ \\
$\delta \pi 1-0$ U3 $\Delta 12 / \mathrm{U} 5 \Delta 12$ & $1.5 \times 10^{6}$ \\
\hline
\end{tabular}

${ }^{2}$ These are the average obtained with two independent constructs, except the double mutant, which was obtained using pooled DNA from two independent constructs.
6). Because the exact nature of + strand priming in Tyl is unknown, a similar sequence comparison at this end cannot be made. However, if the reverse transcript is to be terminally symmetrical, as it is in retroviruses, then a similar juxtaposition of priming site and LTR is expected for the + strand in Tyl reverse transcripts.

The terminal functional group requirements for the reverse transcript indicate that the recombinant intermediate molecule, generated when a Tyl reverse transcript integrates into a target DNA, is identical in overall topology to that formed during bacteriophage $\mathrm{Mu}$ and Tn10 transposition (Craigie and Mizuuchi 1985; Benjamin and Kleckner 1989) and by Moloney MuLV integration (Fujiwara and Mizuuchi 1988; Brown et al. 1989). Thus, all transposons for which this structure has been determined share this property. The small number of nucleotides required for the integration reaction (presumably reflecting the recognition site of IN) is also in line with that determined in retroviral systems (Panganiban and Temin 1983; Colicelli and Goff 1985, 1988).

We attribute the surprising efficiency of the Tyl reaction utilizing artificial substrates to the very small amount of Tyl DNA found in the VLPs. Probably, there are many Ty VLPs that lack Ty DNA but contain the appropriate protein(s) required for transposition. Moreover, the $20 \%$ efficiency of TYA/TYB frameshifting (Clare et al. 1988) and efficient incorporation of p190TYA/TYB, the precursor to Tyl IN protein, into VLPs (Adams et al. 1987; Muller et al. 1987; Youngren et al. 1988) suggest that there are dozens to hundreds of IN proteins per VLP, whereas there should be only one reverse transcript per VLP, if retroviral precedents hold here (Panganiban and Fiore 1988). Finally, many of the Ty1 DNA molecules inside VLPs are heterogeneous in end structure and presumably nonfunctional, suggesting that the inefficiency of reverse transcriptase and/or RNase $H$ activities encoded by Tyl limit transposition frequency. Indeed, the number of Tyl VLPs observed by electron microscopy in cells in which high-level transposition has been induced (hundreds to thousands) far exceeds the number of transposition events observed over a period of several days in those cells [typically one to five per genome (Boeke et al. 1985, 1988)]. In vitro, the activity of Tyl reverse transcriptase is sluggish compared to that of retroviral enzymes (Garfinkel et al. 1985; Mellor et al. 1985). Ty1-encoded RNase $\mathrm{H}$ activity has not yet been directly demonstrated or measured, although it too may be relatively inactive; many of the double-stranded linear molecules isolated from Tyl VLPs are resistant to cleavage by restriction endonucleases (Eichinger and Boeke 1988), consistent with the existence of RNA tracts within many in vivo Tyl re- 


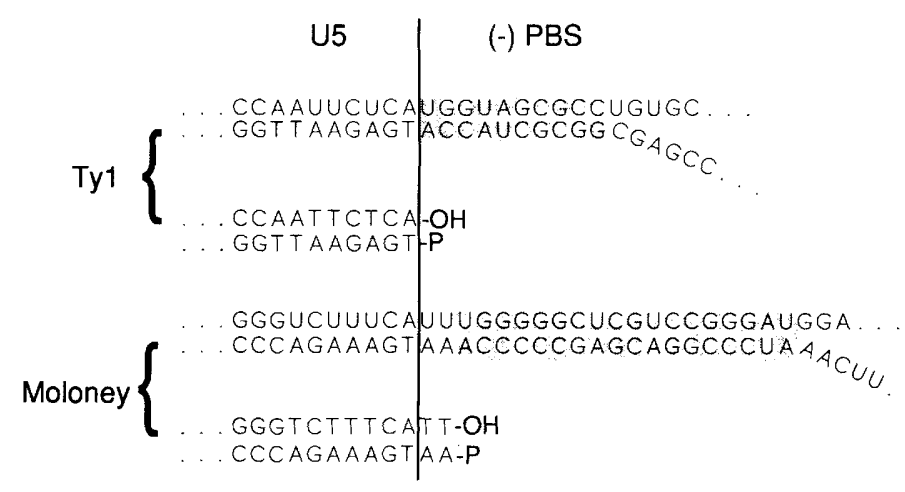

verse transcripts $(M$. Ciriacy, U. Pott, W. Laufer, C. Drewke, and F. Muller, pers. comm.).

\section{Materials and methods}

\section{Plasmid constructions}

$\delta \pi 1$ was constructed as follows. Two oligonucleotides, JB39 (GGTCGACGGATGTTTTATGCATGTTGGAATAGAAATCAA) and JB40 (GGTCGACGGATGTTTTATGCATGAGAATTGGGTGAATGT), were used as primers in a PCR reaction that used a restriction fragment containing the $5^{\prime}$ LTR of Tyl-H3, which contains a XhoI site, as the template. The PCR product of the appropriate size was phenol-extracted and digested with Sall. The SalI PCR fragment was then cloned into the M13mp18 Sall site, to give M138-9. Plasmid man7, digested with SalI, was cloned into the unique $X h o I$ site of $M 13 \delta-9$, to give $M 13 \delta \pi 1$.

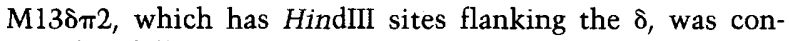

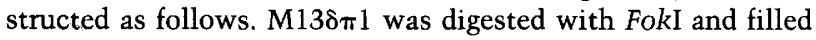
in. The $\delta$-containing FokI fragment was isolated and ligated to M13mp18, which had been digested with HindIII and filled in.

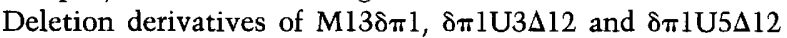
were constructed as described (Kunkel 1985). The oligonucleotides used as primers were JB51 (GCATGTTGGAATAGAGGTCACCAGCTTGCGTTGCTGG) for U3\$12 and IB49 (GTTGAGAAAGAATTCCAAGGACCCAATTCTCATGC) for U5 $\triangle 12$. The double mutant was constructed by remutagenizing $\delta \pi 1 U 3 \Delta 12$ with JB49. The termini of all constructs were sequenced and shown to be exactly as indicated.

\section{Preparation of $\delta \pi$ substrates}

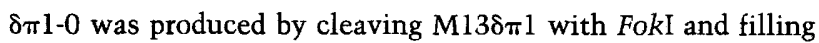

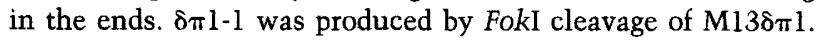
$\delta \pi 1-2$ was produced by cleavage with FokI, filling in in the presence of $\left[\alpha^{-32} \mathrm{P}\right] \mathrm{dCTP}$, electrophoretic purification of the product, filling in in the presence of dATP and dTTP, electrophoretic purification of the product, and filling in with dCTP and $2^{\prime}, 3^{\prime}$ dATP la control aliquot was filled in in the presence of dCTP and dATP and was fully active). $\delta \pi 1-3$ is prepared by treating FokI-digested M138 $\pi 1$ with calf intestine alkaline phosphatase, followed by filling in. An aliquot of the reaction product was tested for phosphate removal by self-ligation; no ligation of $\delta \pi 1-3$ was observed, whereas control $\delta \pi 1-0$ made at the same time was $>90 \%$ ligated. $\delta \pi 1-4$ was produced by NsiI cleavage of

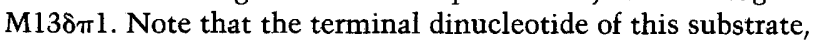
$C A$, is identical to the functional ends of $\delta \pi 1-0 . \delta \pi 2-1$ is pro-

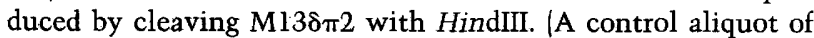
$\delta \pi 2-1$ subsequently treated with S1 nuclease was fully active.)
Figure 6. Relationship of primer binding site and LTR sequences in Tyl and retroviruses. The U5 sequences are separated from the primer binding site sequences by a vertical line. Regions of complementarity between Ty RNA and tRNA $^{\mathrm{i}-\mathrm{Met}}$ (Eibel et al. 1981) and Moloney RNA and tRNA ${ }^{\text {Pro }}$ (Chen and Barker 1984) are shaded. The sequences of Tyl and Moloney murine leukemia virus RNAs and the corresponding $(-)$ strong stop DNAs are shown as the upper double-stranded sequences of each pair. The lower doublestranded sequences are the predicted terminal structures of the right ends of the completed reverse transcripts; in the case of Moloney, the $3^{\prime}$ terminal TT is removed by IN prior to its joining to target DNA. No such reaction is expected to be required for Tyl.
All substrates were agarose gel-purified prior to use in in vitro integration reactions.

\section{$V L P$ isolation and in vitro transposition reactions}

VLPs were first purified over sucrose step gradients, as described (Eichinger and Boeke 1988) using buffer B/EDTA throughout the procedure, followed by centrifugation of the three peak 1.2-ml fractions on a second, linear $15-50 \%$ sucrose gradient. The RT-positive fractions were pooled and concentrated by pelleting at $80,000 \mathrm{~g}$ for $1 \mathrm{hr}$ at $4^{\circ} \mathrm{C}$. Pellets from each $500-\mathrm{ml}$ culture were resuspended in $100 \mu \mathrm{l}$ of $\mathrm{H}_{2} \mathrm{O}$ and stored $\left(-80^{\circ} \mathrm{C}\right)$. In vitro transposition reactions were performed by combining in the following order: $\mathrm{H}_{2} \mathrm{O}$ (to obtain a final volume of $20 \mu 1\}, 2 \mu \mathrm{l}$ of $\delta \pi$ substrate $\{0-25 \mathrm{ng} / \mu \mathrm{l}\}, 2 \mu \mathrm{l}$ of VLP suspension, $2.5 \mu \mathrm{l}$ of $\lambda \mathrm{gtWES} \cdot \lambda \mathrm{B}$ concatemerized DNA (400 ng/ $\mu \mathrm{ll}), 2$ $\mu l(1 \mu \mathrm{g})$ of $10 \times$ transposition buffer, and $5 \mu l$ of $20 \%$ PEG8000. Contents were mixed by gentle tapping and incubated $\left(30^{\circ} \mathrm{C}\right)$ for $30 \mathrm{~min}$ (or as noted otherwise). Subsequent steps [solvent extraction, packaging reactions, etc., were as described (Eichinger and Boeke 1988)]. Total transposition events per reaction were calculated using the formula $\mathrm{A} / \mathrm{B} \cdot \mathrm{C} / \mathrm{D} \cdot \mathrm{E}$, described by Brown et al. (1987). [A = number of plaques on CES200; $\mathrm{B}=$ number of plaques on LE392; $\mathrm{C}=$ number of $\lambda \mathrm{gtWES}$ genomes in $1.0 \mu \mathrm{g}$ DNA $\left(2.2 \times 10^{10}\right) ; \mathrm{D}=$ fraction of the $\lambda \mathrm{gtWES}$ genome tolerant of insertion (0.27); $\mathrm{E}=\mathrm{a}$ correction factor for the differential packaging/plating efficiency between $\lambda g t W E S$ and recombinant phage.]

\section{Acknowledgments}

We are grateful to L. Braiterman for sequencing the termini of some of the $\delta \pi$ substrates. We thank L. Braiterman, K. Chapman, P. Hieter, S. Desiderio, and an anonymous reviewer for helpful criticism of the manuscript. This work was supported by National Institutes of Health (NIH) grant GM36481 (J.D.B.) and NIH postdoctoral training grant CA09139 (D.J.E.).

\section{References}

Adams, S.E., J. Mellor, K. Gull, R.B. Sim, M.F. Tuite, S.M. Kingsman, and A.J. Kingsman. 1987. The functions and relationships of Ty-VLP proteins in yeast reflect those of mammalian proteins. Cell 49: 111-119.

Benjamin, H.W. and N. Kleckner. 1989. Intramolecular transposition by Tn10. Cell 59: 373-383.

Boeke, J.D., D.J. Garfinkel, C.A. Styles, and G.R. Fink. 1985. Ty elements transpose through an RNA intermediate. Cell 40: $491-500$. 
Boeke, J.D., H. Xu, and G.R. Fink. 1988. A general method for the chromosomal amplification of genes in yeast. Science 239: $280-282$.

Bowerman, B., P.O. Brown, J.M. Bishop, and H.E. Varmus. 1989. A nucleoprotein complex mediates the integration of retroviral DNA. Genes Dev. 3: 469-478.

Brown, P.O., B. Bowerman, H.E. Varmus, and J.M. Bishop. 1987. Correct integration of retroviral DNA in vitro. Cell 49: 347-356.

. 1989. Retroviral integration: structure of the initial covalent product and its precursor, and a role for the viral IN protein. Proc. Natl. Acad. Sci. 86: 2525-2529.

Chen, H.R. and W.C. Barker. 1984. Nucleotide sequences of the retroviral long terminal repeats and their adjacent regions. Nucleic Acids Res. 12: 1767-1778.

Clare, J.J., M. Belcourt, and P.J. Farabaugh. 1988. Efficient translational frameshifting occurs within a conserved sequence of the overlap between the two genes of a yeast Tyl transposon. Proc. Natl. Acad. Sci. 85: 6816-6820.

Colicelli, J. and S.P. Goff. 1985. Mutants and pseudorevertants of Moloney murine leukemia virus with alterations at the integration site. Cell 42: 573-580.

- 1988. Sequence and spacing requirements of a retrovirus integration site. J. Mol. Biol. 199: 47-58.

Craigie, R. and K. Mizuuchi. 1985. Mechanism of transposition of bacteriophage $\mathrm{Mu}$ : structure of a transposition intermediate. Cell 41: 867-876.

Eibel, H., J. Gafner, A. Stotz, and P. Philippsen. 1981. Characterization of the yeast mobile genetic element Tyl. Cold Spring Harbor Symp. Quant. Biol. 45: 609-617.

Eichinger, D.J. and J.D. Boeke. 1988. The DNA intermediate in yeast Tyl element transposition copurifies with virus-like particles: Cell-free Tyl transposition. Cell 54: 955-966.

Fujiwara, T. and R. Craigie. 1989. Integration of mini-retroviral DNA: A cell-free reaction for biochemical analysis of retroviral integration. Proc. Natl. Acad. Sci. 86: 3065-3069.

Fujiwara, T. and K. Mizuuchi. 1988. Retroviral DNA integration: structure of an integration intermediate. Cell 54: 497504.

Garfinkel, D.J., J.D. Boeke, and G.R. Fink. 1985. Ty element transposition: Reverse transcriptase and virus-like particles. Cell 42: 507-517.

Kunkel, T.A. 1985. Rapid and efficient site-specific mutagenesis without phenotypic selection. Proc. Natl. Acad. Sci. 82: $488-492$.

Mellor, J., M.H. Malim, K. Gull, M.F. Tuite, S. McCready, T. Dibbayawan, S.M. Kingsman, and A.J. Kingsman. 1985. Reverse transcriptase activity and Ty RNA are associated with virus-like particles in yeast. Nature 318: $583-586$.

Müller, F., K.-H. Bruhl, K. Freidel, K.V. Kowallik, and M. Ciriacy. 1987. Processing of Tyl proteins and formation of Tyl virus-like particles in Saccharomyces cerevisiae. Mol. Gen. Genet. 207: 421 - 429.

Panganiban, A.T. and D. Fiore. 1988. Ordered interstrand and intrastrand DNA transfer during reverse transcription. Science 241: 1064-1069.

Panganiban, A.T. and H.M. Temin. 1983. The terminal nucleotides of retrovinus DNA are required for integration but not virus production. Nature 306: 155-160.

Roth, M.J., P.L. Schwartzberg, and S.P. Goff. 1989. Structure of the termini of DNA intermediates in the integration of retroviral DNA: Dependence on IN function and terminal DNA sequence. Cell 58: 47-54.

Sanger, F., A.R. Coulson, G.F. Hong, D.F. Hill, and G.B. Petersen. 1982. Nucleotide sequence of bacteriophage lambda DNA. I. Mol. Biol. 162: 729-773.
Youngren, S.D., J.D. Boeke, N.J. Sanders, and D.J. Garfinkel. 1988. Functional organization of the retrotransposon $\mathrm{Ty}$ from Saccharomyces cerevisiae: The Ty protease is required for transposition. Mol. Cell. Biol. 8: 1421-1431. 


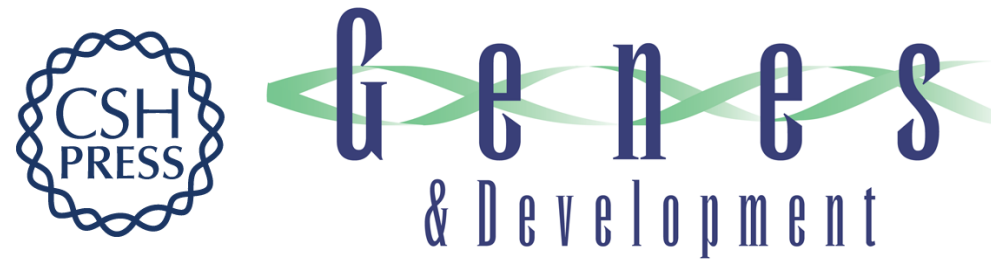

\section{A specific terminal structure is required for Ty1 transposition.}

D J Eichinger and J D Boeke

Genes Dev. 1990, 4:

Access the most recent version at doi:10.1101/gad.4.3.324 $\begin{array}{ll}\text { References } & \text { This article cites } 25 \text { articles, } 9 \text { of which can be accessed free at: } \\ \text { http://genesdev.cshlp.org/content/4/3/324.full.html\#ref-list-1 }\end{array}$

License

Email Alerting Service

Receive free email alerts when new articles cite this article - sign up in the box at the top right corner of the article or click here.

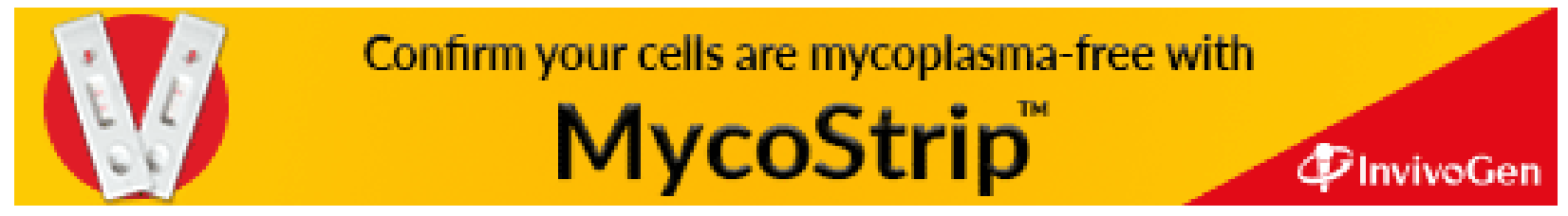

